\title{
Beneficios y riesgos potenciales de las metas intensivas en el tratamiento de la hipertensión arterial. Revisión sistemática y metaanálisis de ensayos clínicos
}

\section{Benefits and potential risks of intensive goals in the treatment of arterial hypertension. Systematic review and meta-analysis of clinical trials}

\author{
Sebastián García-Zamora*, Andrés Rosende, Brunilda Casetta, María F. Grande-Ratti, Natalia Carli, \\ Maria F. Bertarini, María A. Alcuaz y Verónica Schoj
}

Dirección Nacional de Promoción de la Salud y Control de Enfermedades Crónicas No Transmisibles, Ministerio de Salud de la Nación Argentina,Buenos Aires, Argentina

\begin{abstract}
Resumen
Introducción y objetivos: Existe controversia respecto a las metas más adecuadas para el control de la presión arterial. Debido a ello nos propusimos valorar los beneficios y riesgos de adoptar distintos umbrales terapéuticos. Métodos: Revisión sistemática y metaanálisis de ensayos clínicos de grandes dimensiones a fin de valorar el impacto de las distintas estrategias terapéuticas en la reducción de eventos cardiovasculares y desarrollo de efectos adversos serios. Resultados: Se incluyeron cuatro ensayos con 29,820 participantes, con edad media de $65 \pm 7.9$ años. El $42.2 \%$ eran mujeres y el $22 \%$ eran diabéticos. Globalmente las metas intensivas mostraron una tendencia no significativa hacia la reducción de la mortalidad cardiovascular (riesgo relativo [RR]: 0.89; intervalo de confianza del 95\% [IC 95\%]: 0.68-1.07; $p=0.16$ ) sin impacto en la mortalidad total $(p=0.45)$ y con moderada heterogeneidad entre los ensayos (índice I [I]: 44 y 59\% respectivamente). En cambio, sí redujeron eventos cardiovasculares no fatales (RR: 0.82; IC 95\%: 0.70-0.91; $p=0.0003$ ), siendo esto consistente en los ensayos analizados ( $\left.P^{2}: 0 \%\right)$. Respecto a los efectos adversos, las metas intensivas generaron más consultas a guardia 0 internaciones (RR: 1.98; IC 95\%: 1.59-2.46; $p<0.0001 ; 1$ : 14\%), sin claro incremento en la insuficiencia renal (RR: 1.65; IC95\%: 0.942.89) y con aumento de las caídas y síncope (RR: 2.39; IC 95\%: 1.56-3.67; p<0.0001; R: 28\%). Conclusión: Metas intensivas de presión arterial reducen eventos cardiovasculares no fatales, sin impacto en la mortalidad, y con un incremento en el riesgo de eventos adversos. Parecería razonable individualizar los objetivos terapéuticos de acuerdo con el riesgo de cada paciente. Registro: PROSPERO (CRD42020149134).
\end{abstract}

Palabras clave: Hipertensión arterial. Beneficios. Riesgos. Metas intensivas. Metaanálisis.

\begin{abstract}
Introduction and objectives: There is controversy regarding the most appropriate goals for blood pressure control. We assess the benefits and risks of chossing different therapeutic thresholds. Methods: We perform a systematic review and meta-analysis of large clinical trials in order to assess the impact of different therapeutic strategies on the reduction of car-


diovascular events and the development of serious adverse effects. Results: Four trials with 29,820 participants were included, with mean age of $65 \pm 7.9$ years; $42.2 \%$ were women and $22 \%$ were diabetic. Overall, intensive goals showed a non-significant trend towards reducing cardiovascular mortality (relative risk [RR]: 0.89; 95\% confidence interval [Cl]: 0.68-1.07; $p=0.16)$ with non-impact in total mortality $(p=0.45)$ and with moderate heterogeneity among the included trials (I index [I? :44 and 59\% respectively). Nevertheless, intensive goals reduce non-fatal cardiovascular events (RR: 0.82; 95\% Cl: 0.70-0.91; $p=0.0003$ ), being consistent in all the analyzed trials ( $R$ : $0 \%)$. Regarding adverse effects, intensive goals caused more emergency consultations or hospitalization (RR: 1.98; 95\% Cl: 1.59-2.46; $p<0.0001 ; 1$ : 14\%), with no clear increase in renal failure (RR: 1.65; 95\% Cl: 0.94-2.89) but with increase in falls and syncope (RR: 2.39; 95\% Cl: 1.56-3.67; p<0.0001; 1: 28\%). Conclusion: Intensive blood pressure goals reduce non-fatal cardiovascular events, without reduction in mortality, and with an increase of risk of adverse events. These results suggest that individual goals should be set according to the risk of each patient. Clinical trial registration: PROSPERO (CRD42020149134).

Key words: Hypertension. Benefits. Risks. Intensive goals. Meta-analysis.

\section{Introducción}

Aunque la relación entre presión arterial (PA) y eventos vasculares es positiva y lineal desde valores relativamente bajos, establecer un claro punto de corte para el diagnóstico y seguimiento de la hipertensión arterial (HTA) tiene sus ventajas, tanto para los médicos como para los pacientes ${ }^{1}$. Por estas razones, desde finales de la década de 1970 han surgido los primeros consensos para el manejo de esta entidad ${ }^{2,3}$. La IV Declaración Conjunta del Comité Nacional para la detección, evaluación y tratamiento de la HTA (conocidacomo JNC IV por sus siglas en inglés) del año 1988 establecía como puntos de corte para diagnóstico y tratamiento de HTA cifras $\geq 160 / 90 \mathrm{mmHg}$ de presión arterial sistólica (PAS) y diastólica (PAD), respectivamente ${ }^{4}$. En el año 1993 se publicó la JNC V la cual modificó estos valores por cifras $\geq 140 / 90 \mathrm{mmHg}^{5}$, las cuales perduraron sin cuestionamientos hasta años recientes. En noviembre de 2017 se publicó la última guía para el diagnóstico y tratamiento de la HTA elaborada por múltiples sociedades científicas de diferentes especialidades de EE.UU. ${ }^{6}$, la cual estableció un nuevo punto de corte para el diagnóstico de HTA, fijándose dicho valor en 130/80 mmHg. El elemento de mayor peso para impulsar la modificación de las metas fue el ensayo clínico conocido con el acrónimo SPRINT (Systolic Blood Pressure Intervention Trial $)^{7}$. Este estudio recibió numerosos cuestionamientos prácticos ${ }^{8}$ y metodológicos ${ }^{9-11}$, al tiempo que se ha criticado la técnica empleada para la toma de $\mathrm{PA}^{12-15}$. La discusión respecto a las nuevas metas en HTA llevó a que se multipliquen los metaanálisis intentando responder este interrogante ${ }^{16-18}$. Sin embargo, en lugar de saldar el conflicto, las nuevas publicaciones han contribuido a perpetuarlo, ya que los diferentes análisis han mostrado resultados opuestos. Esto se explica fundamentalmente por la heterogeneidad de los criterios de inclusión empleados a la hora de seleccionar los estudios a metaanalizar. Por otra parte, la mayoría de estas publicaciones se han centrado en los beneficios del tratamiento intensivo de la HTA, pero sin ponderar los riesgos que esta intervención supone. Por ello, en el marco del desarrollo de la Guía Nacional de Práctica Clínica para el diagnóstico y tratamiento de la HTA del Ministerio de Salud de la Nación Argentina resolvimos realizar un metaanálisis a fin de ponderar los umbrales terapéuticos más adecuados para el tratamiento de personas con HTA.

\section{Materiales y métodos}

\section{Criterios de inclusión}

Se incluyeron ensayos clínicos aleatorizados (ECA) que se hayan propuesto como objetivo primario comparar metas intensivas y habituales de PA, con al menos 1,000 participantes por grupo, y con seguimiento igual o superior a 12 meses.

Se definieron como metas intensivas de PA valores iguales o menores a $130 / 80 \mathrm{mmHg}$ y metas habituales valores similares o menores a $140 / 90 \mathrm{mmHg}$. Se excluyeron estudios con un diseño no aleatorizado, e informes repetidos de un mismo ECA, si estos no agregaban información a la brindada por la comunicación original del estudio. Asimismo se excluyeron aquellos ECA en los cuales la rama control presentó promedios de cifras tensionales mayores a $145 / 95 \mathrm{mmHg}$. Para la comunicación de la metodología ${ }^{19}$ y los resultados se siguieron las recomendaciones PRISMA ${ }^{20}$.

\section{Búsqueda bibliográfica}

Se realizó una búsqueda de ECA, sin restricciones temporales y utilizando los idiomas inglés, portugués y 
Tabla 1. Características de los estudios incluidos

\begin{tabular}{|c|c|c|c|c|}
\hline Características & $\begin{array}{c}\text { ACCORD } \\
\text { ACCORD Study Group, } \\
2010^{26}\end{array}$ & $\begin{array}{l}\text { SPS3 } \\
\text { SPS3 Study Group, } \\
2013^{28}\end{array}$ & $\begin{array}{l}\text { SPRINT } \\
\text { SPRINT Research } \\
\text { Group, 20157 }\end{array}$ & $\begin{array}{l}\text { HOPE-3 } \\
\text { Lonn, et al., } \\
2016^{27}\end{array}$ \\
\hline Año de publicación & 2010 & 2013 & 2015 & 2016 \\
\hline Participantes (n) & 4,733 & 3,020 & 9,361 & 12,705 \\
\hline Edad (DE) & $62.2 \pm 6.9$ & $63 \pm 10.8$ & $67.9 \pm 9.5$ & $65.8 \pm 6.4$ \\
\hline PA ingreso $(\mathrm{mmHg})$ & $139 / 76$ & $143 / 79$ & $140 / 78$ & $138 / 82$ \\
\hline Mujeres (\%) & $2,258(47.7)$ & $1,118(37)$ & $3,332(35.6)$ & $5,874(46.2)$ \\
\hline Diabéticos (\%) & $4,733(100)$ & $1,106(36.6)$ & $0(0)$ & $731(5.8)$ \\
\hline Índice de masa corporal $\left(\mathrm{kg} / \mathrm{m}^{2}\right)$ & $32.1 \pm 5.6$ & $29.2 \pm 6.8$ & $29.9 \pm 5.8$ & $27.1 \pm 4.8$ \\
\hline Enfermedad coronaria (\%) & \multirow[t]{2}{*}{$33.7 \% *$} & $11 \%$ & $20.1 \%$ & $0 \%$ \\
\hline Enfermedad cerebrovascular (\%) & & $100 \%$ & $0 \%$ & $0 \%$ \\
\hline Metas de PA en el estudio & PAS $<120$ & PAS $<130$ & PAS $<120$ & Dosis fija de fármaco \\
\hline Diferencia de PA al final del seguimiento $(\mathrm{mmHg})$ & $14.2 / 6.1$ & $11 /-^{\dagger}$ & $14.8 / 7.6$ & $6 / 3$ \\
\hline Seguimiento promedio (años) & 4.7 & 3.7 & $3.26^{\ddagger}$ & 5.6 \\
\hline Tipo de estudio & Abierto & Abierto & Abierto & Doble ciego \\
\hline $\begin{array}{l}\text { Riesgo cardiovascular estimado de los pacientes } \\
\text { incluidos }\end{array}$ & Alto & Alto & Alto & Intermedio \\
\hline $\begin{array}{l}\text { *No se encuentra discriminado enfermedad coronaria y cereb } \\
{ }^{+} \text {No se encuentra informado el valor de PAD. } \\
\text { †Detenido prematuramente por beneficio. } \\
\text { PA: presión arterial; DE: desviación estándar; PAS: presión art } \\
\text { Small Subcortical Strokes; SPRINT: Systolic Blood Pressure Ir }\end{array}$ & ar & & & \\
\hline
\end{tabular}

español. Dicho proceso fue realizada en simultáneo por dos operadores experimentados (S.G.Z. y A.R.), quienes seleccionaron los estudios a incluir. Las diferencias fueron resueltas por consenso. Se emplearon como motores de búsqueda Medline, LILACS, Google académico y la base de datos de la colaboración Cochrane.

\section{Puntos finales evaluados}

De los estudios seleccionados se recabó la ocurrencia de mortalidad total, mortalidad cardiovascular y eventos cardiovasculares: síndromes coronarios agudos (agrupando infarto agudo de miocardio [IAM] con y sin elevación del segmento ST y angina inestable), accidentes cerebrovasculares(ACV) isquémicos y hemorrágicos, y accidente isquémico transitorio. Como efectos adversos se valoraron: caídas, síncope, hipotensión arterial, insuficiencia renal aguda y los efectos adversos "serios", entendidos como tal todos aquellos eventos que ocasionaron una consulta a sala de emergencias o guardia, o que requirieron la internación de los participantes, independientemente de que los ECA hayan utilizado otra definición operativa para estos eventos. Para la identificación de estos puntos finales se utilizaron las definiciones previstas por los investi-

\section{Evaluación del riesgo de sesgo}

Se llevó adelante una evaluación del riesgo de sesgo de los estudios incluidos, de acuerdo con las recomendaciones del Manual Cochrane ${ }^{21,22}$. Los dominios evaluados fueron: generación de secuencia aleatoria, asignación oculta, cegamiento del personal y los participantes, cegamiento de los evaluadores, evaluación incompleta de datos de resultado, reporte selectivo y un último dominio para otros sesgos potenciales. El riesgo de sesgo fue valorado de forma categórica en: bajo riesgo, riesgo incierto y alto riesgo siguiendo las recomendaciones para tal fin ${ }^{21-23}$.

\section{Registro}

El presente metaanálisis fue registrado en PROSPERO con el número de identificación: CRD42020149134.

\section{Análisis estadístico}

Por tratarse de ensayos clínicos se empleó como medida sumaria riesgos relativos (RR) y sus respectivos intervalos de confianza (IC). Para metaanalizar los datos, y debido a las diferencias de los estudios seleccionados, se optó por un modelo de efectos aleatorios, 
Tabla 2. Características adicionales de los estudios incluidos

\begin{tabular}{|c|c|}
\hline Estudio & Detalles \\
\hline $\begin{array}{l}\text { ACCORD } \\
\text { ACCORD Study Group, } 2010^{26}\end{array}$ & - Hemoglobina glucosilada promedio de los participantes: $8.3 \pm 1.1 \%$ (sin diferencias entre grupos) \\
\hline $\begin{array}{l}\text { SPS3 } \\
\text { SPS3 Study Group, } 2013^{28}\end{array}$ & $\begin{array}{l}\text { - El } 98.6 \% \text { de los participantes fueron enrolados por un accidente cerebrovascular isquémico y el } 1.4 \% \\
\text { por un accidente isquémico transitorio }\end{array}$ \\
\hline $\begin{array}{l}\text { SPRINT } \\
\text { SPRINT Research Group, } 2015^{7}\end{array}$ & $\begin{array}{l}\text { - Detenido prematuramente por beneficio } \\
\text { - Se empleó una estrategia no habitual de toma de PA } \\
\text { - En la rama control al } 87 \% \text { de los participantes se les redujo el tratamiento antihipertensivo que } \\
\text { realizaban, encontrándose los participantes asintomáticos } \\
\text { - Existió significativamente mayor proporción de uso de diuréticos en la rama intensiva }\end{array}$ \\
\hline $\begin{array}{l}\text { HOPE-3 } \\
\text { Lonn, et al., } 2016^{27}\end{array}$ & $\begin{array}{l}\text { - Dosis fija de candesartán } 16 \mathrm{mg} \text { + hidroclorotiazida } 12.5 \mathrm{mg} \\
\text { - Un análisis de subgrupos preespecificado encontró beneficios con la reducción de PA entre los } \\
\text { participantes con mayores valores de PA (tercio superior de la muestra) }\end{array}$ \\
\hline
\end{tabular}

PA: presión arterial; ACCORD: Action to Control Cardiovascular Risk in Diabetes; SPS3: Secondary Prevention of Small Subcortical Strokes; SPRINT: Systolic Blood Pressure Intervention Trial; HOPE-3: Heart Outcomes Prevention Evaluation-3.

como describieron Dersimonian y Laird ${ }^{24}$. Se evaluó la heterogeneidad de estos mediante el cálculo de la varianza entre los estudios (tau cuadrada) y el índice I cuadrado $\left(I^{2}\right)^{25}$. El análisis de datos fue realizado empleando Review Manager 5.3.

\section{Resultados}

Se identificaron 530 artículos potencialmente relevantes, de los cuales se excluyeron 246 por encontrarse duplicados, cinco por no ser ECA y 275 por no cumplir los criterios de inclusión. Finalmente se analizaron cuatro ensayos clínicos (Fig. supl. 1): SPRINT7', ACCORD (Action to Control Cardiovascular Risk in Diabetes) ${ }^{26}$, HOPE-3 (Heart Outcomes Prevention Evaluation-3) ${ }^{27}$ y SPS3 (Secondary Prevention of Small Subcortical Strokes) ${ }^{28}$, con un total de 29.820 participantes. La edad media de los participantes de los estudios fue $65 \pm 7.9$ años; el $42.2 \%$ eran mujeres y el $22 \%$ eran diabéticos. El resto de las características de Ios ECA incluidos se resumen en la tabla 1. Algunas características adicionales relevantes de los ensayos clínicos individuales incluidos en la revisión se encuentran puntualizadas en la tabla 2. Se procedió a valorar la calidad de la evidencia de los estudios de acuerdo con los lineamientos antes mencionados (Fig. supl. 2). En el anexo I se detallan los motivos por los cuales se consideró que algún dominio de cada ensayo poseía un riesgo de sesgo incierto 0 alto.

La figura suplementaria 3 esquematiza la calidad global de la evidencia; se observa que el dominio con mayor riesgo de sesgo es "Cegamiento del personal y los participantes", ya que tres de los cuatro ensayos fueron abiertos.
Analizados globalmente los cuatro estudios (SPRINT, HOPE-3, ACCORD y SPS3) mostraron una tendencia no significativa hacia una reducción de la mortalidad cardiovascular con el empleo de metas intensivas de PA (RR: 0.89; IC95\%: 0.68-1.07; p=0.16), con moderada heterogeneidad (I2: 44\%) (Fig. 1).

Las metas intensivas tampoco redujeron la mortalidad total (RR: 0.94; IC95\%: 0.8-1.1; p=0.45) (Fig. 2).

$\mathrm{Al}$ analizar los eventos cardiovasculares en su conjunto, las metas intensivas los redujeron de forma significativa (RR: 0.82; IC95\%: 0.70-0.91; $p=0.0003$ ), sin evidencia de heterogeneidad (12: 0\%) (Fig. 3).

A fin de valorar adecuadamente la relación riesgo-beneficio de las diferentes metas de control de PA se analizaron los efectos adversos ocurridos en las diferentes ramas de tratamiento. Un estudio (HOPE-3) no reportó la necesidad de atenciones no programadas por parte de los participantes del ensayo debido al empleo de tratamiento farmacológico, y por tanto no pudo ser incluido para tal fin. En los restantes tres estudios los eventos adversos serios fueron más frecuentes en la rama intensiva, siendo esto más notorio en aquellos ECA que fijaron metas más bajas de PA (ACCORD y SPRINT). Globalmente las metas intensivas se asociaron a un riesgo incrementado de eventos adversos serios (RR: 1.98; IC95\%: 1.59-2.46; p<0.0001; I2: 14\%) (Fig. 4).

Debido a que la insuficiencia renal constituye una preocupación mayor a la hora de fijar metas más exigentes de control de PA, se analizó esto como desenlace independiente. Se observó una tendencia no significativa hacia un mayor riesgo en los episodios de deterioro de la función renal con las metas intensivas 
Arch Cardiol Mex. 2020;90(4)

\begin{tabular}{|c|c|c|c|c|c|c|c|c|c|c|}
\hline \multirow{3}{*}{$\begin{array}{l}\text { Study or Subgroup } \\
\text { ACCORD } 2010\end{array}$} & \multicolumn{2}{|c|}{ Metas intensivas } & \multicolumn{2}{|c|}{ Metas habituales } & \multirow[b]{2}{*}{ Weight } & \multirow{2}{*}{$\begin{array}{c}\text { Risk Ratio } \\
\text { M-H, Random, } 95 \% \mathrm{Cl}\end{array}$} & \multirow{2}{*}{\multicolumn{4}{|c|}{$\begin{array}{c}\text { Risk Ratio } \\
\text { M-H, Random, } 95 \% \mathrm{Cl}\end{array}$}} \\
\hline & \multirow{2}{*}{$\begin{array}{r}\text { Events } \\
150\end{array}$} & \multirow{2}{*}{$\begin{array}{l}\text { Total } \\
2363\end{array}$} & \multirow{2}{*}{$\begin{array}{r}\text { Events } \\
144\end{array}$} & \multirow{2}{*}{$\begin{array}{l}\text { Total } \\
2371\end{array}$} & & & & & & \\
\hline & & & & & $23.4 \%$ & $\begin{array}{c}\text { M-H, Random, } 95 \% \mathrm{Cl} \\
1.05[0.84,1.30]\end{array}$ & & & + & \\
\hline HOPE-3 & 342 & 6356 & 349 & 6349 & $31.6 \%$ & $0.98[0.85,1.13]$ & & & & \\
\hline SPRINT 2015 & 155 & 4678 & 210 & 4683 & $25.2 \%$ & $0.74[0.60,0.91]$ & & & & \\
\hline SPS-3 & 106 & 1501 & 101 & 1519 & $19.8 \%$ & $1.06[0.82,1.38]$ & & & 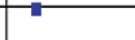 & \\
\hline Total $(95 \% \mathrm{Cl})$ & & 14898 & & 14922 & $100.0 \%$ & $0.94[0.80,1.10]$ & & & & \\
\hline Total events & 753 & & 804 & & & & & & & \\
\hline \multicolumn{7}{|c|}{ Heterogeneity: $\mathrm{Tau}^{2}=0.02 ; \mathrm{Chi}^{2}=7.38, \mathrm{df}=3(P=0.06) ; \mathrm{I}^{2}=59 \%$} & 0.7 & 0.85 & 1.2 & 1. \\
\hline \multicolumn{7}{|c|}{ Test for overall effect: $Z=0.75(P=0.45)$} & & intensivas & Metas habit & \\
\hline
\end{tabular}

Figura 1. Forest plot 1: mortalidad total.

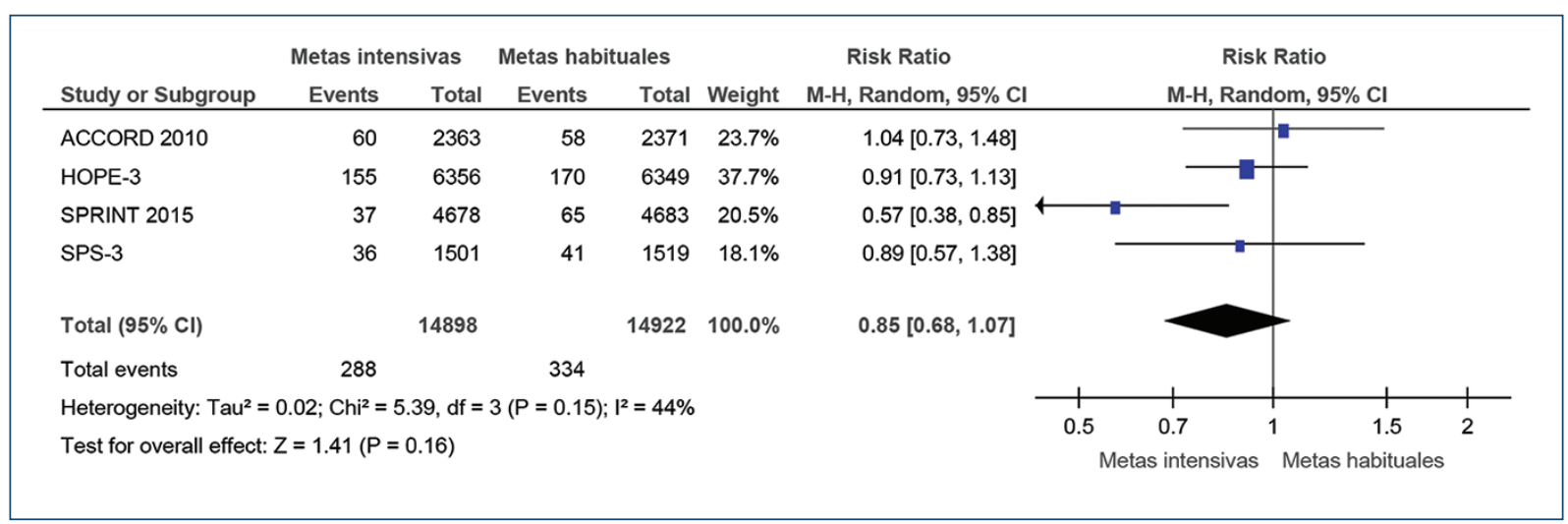

Figura 2. Forest plot 2: mortalidad cardiovascular.

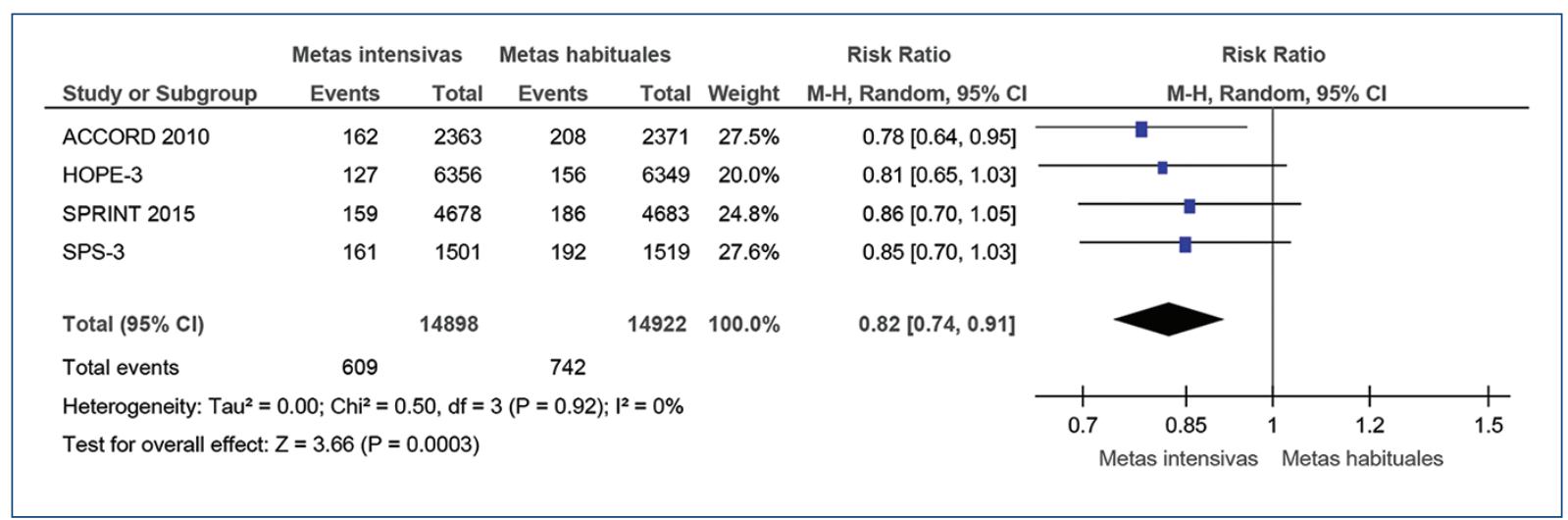

Figura 3. Forest Plot 3: eventos cardiovasculares.

$(p=0.08)$, pero con marcada heterogeneidad entre los ensayos analizados (Fig. 5).

Debe remarcarse que la heterogeneidad respecto al riesgo de deterioro de la función renal no fue solamente estadística, sino que existieron marcadas diferencias en las definiciones clínicas. Así, mientras que en el ACCORD esto fue categorizado como valores de creatinina mayores a $3.3 \mathrm{mg} / \mathrm{dl}$ o necesidad de diálisis, en el SPRINT fue definido como la codificación dentro de los tres primeros diagnósticos al egreso hospitalario. 


\begin{tabular}{|c|c|c|c|c|c|c|c|c|c|}
\hline \multirow{3}{*}{$\begin{array}{l}\text { Study or Subgroup } \\
\text { ACCORD } 2010\end{array}$} & \multicolumn{2}{|c|}{ Metas intensivas } & \multicolumn{2}{|c|}{ Metas habituales } & \multirow[b]{2}{*}{ Weight } & \multirow{2}{*}{$\begin{array}{c}\text { Risk Ratio } \\
\text { M-H, Random, } 95 \% \mathrm{Cl}\end{array}$} & \multirow{2}{*}{\multicolumn{3}{|c|}{$\begin{array}{c}\text { Risk Ratio } \\
\text { M-H, Random, } 95 \% \mathrm{Cl}\end{array}$}} \\
\hline & \multirow{2}{*}{$\begin{array}{r}\text { Events } \\
77\end{array}$} & \multirow{2}{*}{$\begin{array}{l}\text { Total } \\
2363\end{array}$} & \multirow{2}{*}{$\begin{array}{r}\text { Events } \\
30\end{array}$} & \multirow{2}{*}{$\begin{array}{l}\text { Total } \\
2371\end{array}$} & & & & & \\
\hline & & & & & $24.1 \%$ & $2.58[1.70,3.91]$ & & \multirow{2}{*}{\multicolumn{2}{|c|}{$\vec{\square}$}} \\
\hline SPRINT 2015 & 220 & 4678 & 118 & 4683 & $64.9 \%$ & $1.87[1.50,2.33]$ & & & \\
\hline SPS-3 & 23 & 1501 & 15 & 1519 & $10.9 \%$ & $1.55[0.81,2.96]$ & & & \\
\hline Total $(95 \% \mathrm{Cl})$ & & 8542 & & 8573 & $100.0 \%$ & $1.98[1.59,2.46]$ & & & \\
\hline Total events & \multicolumn{2}{|l|}{320} & 163 & & & & & & \\
\hline \multicolumn{7}{|c|}{ Heterogeneity: $\mathrm{Tau}^{2}=0.01 ; \mathrm{Chi}^{2}=2.33, \mathrm{df}=2(P=0.31) ; \mathrm{I}^{2}=14 \%$} & $0.5 \quad 0.7$ & $1 \quad 1.5$ & 2 \\
\hline \multicolumn{7}{|c|}{ Test for overall effect: $Z=6.06(P<0.00001)$} & Metas intensivas & Metas habit & ituales \\
\hline
\end{tabular}

Figura 4. Forest plot 4: eventos adversos serios.

\begin{tabular}{|c|c|c|c|c|c|c|c|}
\hline \multirow[b]{2}{*}{ Study or Subgroup } & \multicolumn{2}{|c|}{ Metas intensivas } & \multicolumn{2}{|c|}{ Metas habituales } & \multirow[b]{2}{*}{ Weight } & \multirow{2}{*}{$\begin{array}{c}\text { Risk Ratio } \\
\text { M-H, Random, } 95 \% \mathrm{Cl}\end{array}$} & \multirow{2}{*}{$\begin{array}{c}\text { Risk Ratio } \\
\text { M-H, Random, } 95 \% \mathrm{Cl}\end{array}$} \\
\hline & Events & Total & Events & Total & & & \\
\hline ACCORD 2010 & 64 & 2363 & 59 & 2371 & $35.9 \%$ & $1.09[0.77,1.54]$ & - \\
\hline HOPE-3 & 32 & 6356 & 20 & 6349 & $29.5 \%$ & $1.60[0.92,2.79]$ & \\
\hline SPRINT 2015 & 88 & 4678 & 34 & 4683 & $34.6 \%$ & $2.59[1.75,3.84]$ & \\
\hline Total $(95 \% \mathrm{Cl})$ & & 13397 & & 13403 & $100.0 \%$ & $1.65[0.94,2.89]$ & \\
\hline Total events & 184 & & 113 & & & & \\
\hline \multicolumn{7}{|c|}{ Heterogeneity: $\mathrm{Tau}^{2}=0.20 ; \mathrm{Chi}^{2}=10.47, \mathrm{df}=2(\mathrm{P}=0.005) ; \mathrm{I}^{2}=81 \%$} & $\begin{array}{ccccc} & 1 & & 1 & 1 \\
0.5 & 0.7 & 1 & 1.5 & 2\end{array}$ \\
\hline \multicolumn{7}{|c|}{ Test for overall effect: $Z=1.74(P=0.08)$} & Metas intensivas Metas habitt \\
\hline
\end{tabular}

Figura 5. Forest plot 5: insuficiencia renal aguda.

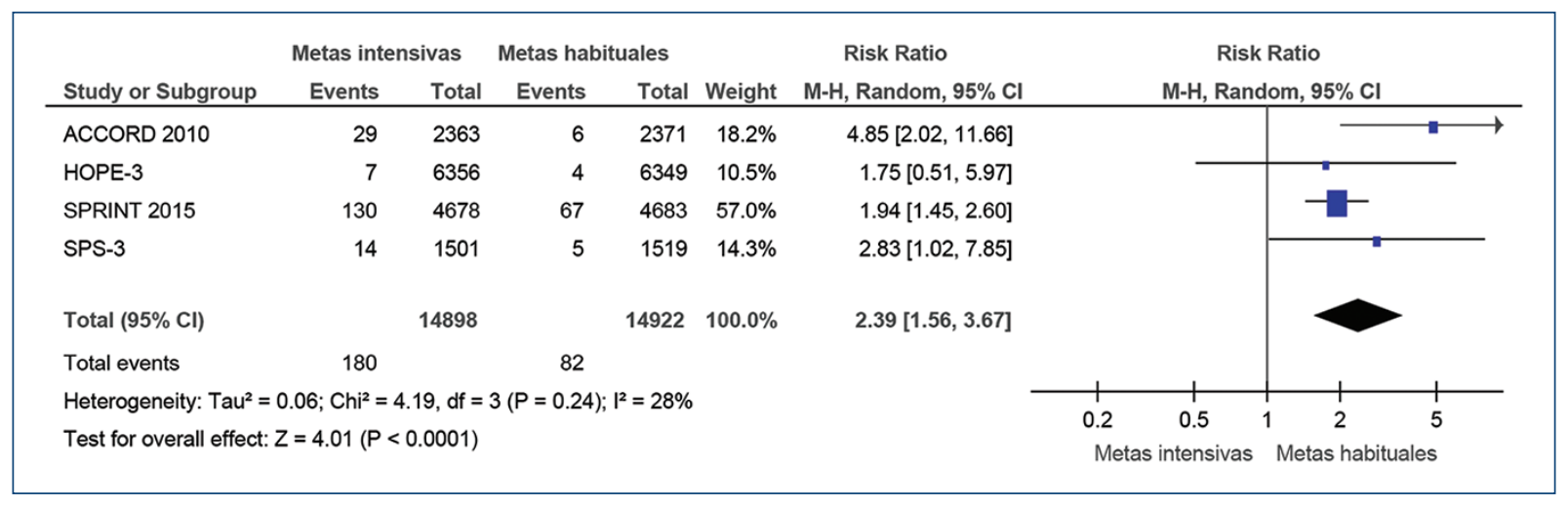

Figura 6. Forest plot 6: caídas y síncope.

Por último se comparó la ocurrencia de síncopes y caídas atribuibles al tratamiento farmacológico, observando un incremento del riesgo en el grupo de metas intensivas (RR: 2.39; IC95\%: 1.56-3.67; $p<0.0001)$, con baja heterogeneidad (I2: 28\%) (Fig. 6).
Similar a lo observado en el análisis de los efectos adversos graves, puede apreciarse cómo los estudios con metas más bajas de PA (ACCORD y SPRINT vs. HOPE-3 y SPS3) aportaron la mayor cantidad de eventos para este desenlace. 
A fin de valorar el impacto de los pacientes diabéticos en estos resultados se llevó a cabo un análisis de sensibilidad excluyendo el estudio ACCORD, sin encontrar diferencias a excepción del riesgo de insuficiencia renal, lo cual se tornó significativo (datos no mostrados).

Si bien la evaluación del sesgo de publicación tiene limitaciones en contexto de metaanálisis con menos de 10 estudios $^{21,22}$, esta se realizó, no sugiriendo sesgos importantes para la presente revisión (Fig. supl. 4).

\section{Discusión}

Nuestro metaanálisis sugiere que las metas intensivas de control de PA reducen eventos cardiovasculares mayores, con una tendencia no significativa hacia la reducción de mortalidad cardiovascular y total, y un incremento en el riesgo de efectos adversos. Si bien los beneficios del tratamiento antihipertensivo se ha demostrado hace décadas ${ }^{29}$, en los últimos años los resultados del estudio SPRINT ${ }^{7}$ y el cambio en las cifras tensionales para diagnosticar HTA de la última Guía multisociedades de EE.UU. para el manejo de esta patología ${ }^{6}$ han generado notable controversia respecto a las metas terapéuticas más beneficiosas. Así algunos metaanálisis han respaldado el cambio de objetivos terapéuticos, planteando que cifras tensionales menores a $130 \mathrm{mmHg}$ de PAS tendrían beneficios en eventos cardiovasculares y reducirían la mortalidad de los pacientes tratados ${ }^{30-34}$. Al analizar los resultados de estos metaanálisis se deben tener presentes cuatro aspectos: a) incluyeron estudios con pacientes con un amplio rango de valores de PA (incluso por encima de $180 \mathrm{mmHg}$ ) y con objetivos terapéuticos que variaron desde menos de $120 \mathrm{mmHg}$ hasta menos de $160 \mathrm{mmHg}$ de PAS; b) incluyeron estudios de pequeñas dimensiones (inclusive menos de 250 participantes); c) algunos de los estudios incluidos tenían como objetivos primarios puntos finales subrogados (como progresión de la disfunción renal) sin haber evaluado desenlaces fatales, y d) incluyeron estudios que no comunicaron efectos adversos del tratamiento, o que se limitaron a informar del porcentaje de pacientes que abandonaron el tratamiento por estos. El único ECA que encontró reducción en la mortalidad cardiovascular y total con metas intensivas de PA fue SPRINT, pero llamativamente esto ocurrió sin demostrar diferencias en la ocurrencia de IAM y ACV9-15.

Debido a que los efectos adversos de los fármacos en ocasiones ocurren tardíamente, y que el cálculo del tamaño muestral de los ECA se hace para detectar los componentes del punto final primario, pero no los efectos adversos, estos suelen estar subrepresentados en la mayoría de los ensayos. Sumando el no reporte de estos, existe el riesgo de sobrevalorar la intervención, magnificando los beneficios y omitiendo las consecuencias indeseables asociadas a esta.

Dos metaanálisis ${ }^{35,36}$ del año 2014 encontraron que si bien la reducción de la PA producía beneficios similares en todos los grupos de participantes, aquellos con mayor riesgo basal experimentaban también mayor reducción absoluta en los eventos. Otro metaanálisis reciente $^{37}$ encontró resultados similares: a iguales descensos en la PA, los pacientes de mayor riesgo cardiovascular obtenían mayores beneficios en términos absolutos. Por tanto, incluir estudios donde los participantes presentaban valores muy elevados de PA al ingreso, o en los cuales la rama control tenía objetivos terapéuticos menos estrictos que los actualmente recomendados (<140/90 $\mathrm{mmHg}$ ) podría ser una fuente potencial de sesgos a la hora de valorar el beneficio de dos metas de PA. Esto motivó que en el presente metaanálisis se hayan incluido únicamente ECA en donde los participantes del grupo control tuvieron valores promedio de PAS con cifras de 140 a $145 \mathrm{mmHg}$. Por otro lado, en patologías de alta prevalencia los estudios de pequeñas dimensiones conllevan dos riesgos: a) que se traten de subpoblaciones particulares que no representen adecuadamente el universo de individuos con la enfermedad, y b) que exista sesgo de publicación para estudios positivos. A fin de evitar estas fuentes de error se incluyeron solamente estudios de medianas o grandes dimensiones y con seguimiento promedio de al menos 12 meses.

Tres metaanálisis recientes encontraron resultados similares a los nuestros ${ }^{16,38,39}$. Saiz, et al. ${ }^{16}$ incluyeron seis estudios con 9,795 pacientes y comunicaron que las metas intensivas redujeron eventos cardiovasculares, hospitalización y muerte por insuficiencia cardiaca, sin reducir la mortalidad total o cardiovascular. No encontraron un incremento en la tasa de eventos adversos mayores en el grupo asignado a tratamiento intensivo, pero sí más abandono de medicación por efectos adversos en general. Xie, et al. ${ }^{38}$ incluyeron 19 estudios con 44,989 participantes, encontrando que las metas intensivas de PA reducirían eventos cardiovasculares, albuminuria y la progresión de retinopatía, sin impacto en la mortalidad total, la mortalidad cardiovascular, la insuficiencia cardiaca y la progresión a enfermedad renal terminal. Respecto a los efectos adversos, 
no encontraron un aumento significativo en los efectos adversos serios, pero sí en la ocurrencia de hipotensión grave. Brunström y Carlberg ${ }^{39}$ incluyeron 74 estudios con 306,273 participantes. De acuerdo con sus resultados el impacto en la reducción de eventos variaría según los niveles de PA basales: en los estudios donde los participantes tenían valores de PAS mayores a 160 $\mathrm{mmHg}$ el tratamiento antihipertensivo redujo eventos cardiovasculares y muerte, mientras que en los estudios con cifras basales entre 140 y $159 \mathrm{mmHg}$ el tratamiento solo redujo eventos cardiovasculares. En cambio en los estudios donde los participantes presentaban valores basales de PA menores a $140 \mathrm{mmHg}$, el tratamiento antihipertensivo solo redujo eventos cardiovasculares cuando los sujetos tenían historia de enfermedad coronaria. En el presente estudio se analizaron con mayor profundidad la ocurrencia de distintos eventos adversos, entendiendo que la elección de metas antihipertensivas habituales e intensivas debiera ser una decisión individualizada, sopesando los beneficios y riesgos potenciales, con resultados que avalan las recomendaciones de la Sociedad Europea de Cardiología ${ }^{40}$.

Algunos de los metaanálisis antes mencionados incluyeron entre los eventos cardiovasculares la ocurrencia de insuficiencia cardiaca con las distintas metas de PA. De hecho en SPRINT la insuficiencia cardiaca fue uno de los componentes del punto final primario donde se observó mayor diferencia entre grupos ${ }^{7}$. Sin embargo, tanto en este estudio como en otros los grupos con tratamiento intensivo recibieron mayor proporción de diuréticos ${ }^{12}$. Debido a esto resulta complejo determinar si las metas intensivas de PA reducen per se la ocurrencia de insuficiencia cardiaca o si esta observación se encuentra confundida por el tratamiento farmacológico.

En nuestro metaanálisis el $22 \%$ de los pacientes incluidos $(n=6,570)$ eran diabéticos. De estos, la mayor proporción provienen del estudio ACCORD, el cual fue realizado exclusivamente entre individuos con esta afección. Si bien ACCORD no mostró beneficios en el punto final primario analizado, tampoco aportó indicios de un peor pronóstico con metas intensivas. Al excluir a los participantes de este estudio de nuestro análisis, no encontramos diferencias sustanciales en los resultados. Esto hace suponer que los resultados generales de nuestro análisis muy probablemente puedan aplicarse a los pacientes con esta enfermedad.

Finalmente otro aspecto debatible es incluir en el presente metaanálisis estudios con pacientes de riesgo cardiovascular alto e intermedio. Si aceptamos que la
PA es una variable continua y que, por tanto, su relación con el riesgo cardiovascular es lineal, uno esperaría que el impacto en su reducción sea similar pero de menor magnitud en individuos con menor riesgo. Esto se observa en nuestro metaanálisis con los resultados de HOPE-3. Si bien esto podría haber disminuido el estimador global de los efectos analizados, aumenta la validez externa de estos, ya que en la práctica cotidiana no siempre resulta tan sencillo estimar con precisión el riesgo futuro de cada persona individual.

\section{Limitaciones}

La primer gran limitación del presente trabajo es no disponer de las bases de datos de los estudios incluidos. No realizar un metaanálisis de datos individuales impide llevar adelante algunos análisis de sensibilidad, como una valoración más pormenorizadade los pacientes diabéticos o delinear mejor las características de los sujetos más susceptibles a desarrollar efectos adversos con las metas intensivas de control de PA. En segundo lugar, si bien utilizar criterios de inclusión rigurosos limitan algunos sesgos ya mencionados, conllevan riesgo de incurrir en otros, como sesgo de selección. De hecho, nuestro metaanálisis es el que posee menor número de estudios incluidos entre los recientemente realizados. Sin embargo, al incluir estudios de grandes dimensiones posee un número de participantes superior al de otras publicaciones. Además de lo antes mencionado, debe tenerse presente que estos datos no son aplicables a pacientes octogenarios, por ser una población francamente subrepresentada en la mayoría de los ensayos clínicos. De hecho, el único estudio disponible hasta la fecha en estos pacientes fijó metas de PAS de $150 \mathrm{mmHg}$. Debido a que el horizonte temporal de los ensayos clínicos es limitado, no es posible determinar el impacto a mediano o largo plazo que podría tener optar por metas intensivas o conservadoras de PA. Si bien es dable suponer que la reducción de eventos no fatales podría tener un impacto beneficioso en la disminución de eventos fatales a largo plazo, no es posible respaldar esta inferencia con los datos disponibles actualmente. Por otro lado, esta limitación es aplicable tanto a los beneficios como al riesgo de desarrollo de eventos indeseables en el seguimiento.

\section{Conclusión}

Nuestros datos sugieren que las metas intensivas de PA con valores de presión sistólica próximos a 130 
$\mathrm{mmHg}$ reducen los eventos cardiovasculares, sin impacto en la mortalidad a corto plazo, y con un incremento en el riesgo de eventos adversos. Asimismo, no encontramos evidencia que sugiera la necesidad de fijar metas de control de presión diferentes en individuos diabéticos y no diabéticos.

Debido a ello se debe reforzar el control de la PA para todos los sujetos hipertensos, considerando emplear metas intensivas en aquellos individuos que pertenezcan a subgrupos de mayor riesgo cardiovascular, con bajo riesgo de efectos adversos.

Nuevos estudios son necesarios para evaluar el impacto a mediano y largo plazo de las distintas metas antihipertensivas.

\section{Financiamiento}

La presente investigación no ha recibido ninguna beca específica de agencias de los sectores público, comercial o sin ánimo de lucro.

\section{Conflictos de intereses}

Los autores declaran no tener conflicto de intereses.

\section{Responsabilidades éticas}

Protección de personas y animales. Los autores declaran que para esta investigación no se han realizado experimentos en seres humanos ni en animales.

Confidencialidad de los datos. Los autores declaran que en este artículo no aparecen datos de pacientes.

Derecho a la privacidad y consentimiento informado. Los autores declaran que en este artículo no aparecen datos de pacientes.

\section{Material suplementario}

El material suplementario se encuentra disponible en Archivos de Cardiología de México (http://www.archivoscardiologia.com/). Este material es provisto por el autor correspondiente y publicado online para beneficio del lector. El contenido del material suplementario es responsabilidad de los autores.

\section{Bibliografía}

1. Kotchen TA. Developing hypertension guidelines: an evolving process. Am J Hypertens.2014;27:765-72.

2. Report of the Joint National Committee on Detection, Evaluation, and Treatment of High Blood Pressure. A cooperative study. JAMA.1977; 237:255-61.
3. The 1980 report of the Joint National Committee on Detection, Evaluation, and Treatment of High Blood Pressure. Arch Intern Med.1980; 140:1280-5.

4. The 1988 report of the Joint National Committee on Detection, Evaluation, and Treatment of High Blood Pressure. Arch Intern Med.1988; 148:1023-38.

5. The fifth report of the Joint National Committee on Detection, Evaluation, and Treatment of High Blood Pressure (JNC V). Arch Intern Med.1993; 153:154-83.

6. Whelton PK, Carey RM, Aronow WS, Casey DE Jr, Collins KJ, Dennison Himmelfarb C, et al. 2017 ACC/AHA/AAPA/ABC/ACPM/AGS/apha/ASH/ ASPC/NMA/PCNA Guideline for the Prevention, Detection, Evaluation, and Management of High Blood Pressure in Adults: Executive Summary: A Report of the American College of Cardiology/American Heart Association Task Force on Clinical Practice Guidelines. Hypertension.2018;71:1269-324.

7. SPRINT Research Group, Wright JT Jr, Williamson JD, Whelton PK, Snyder JK, Sink KM, Rocco MV, et al. A Randomized trial of intensive versus standard blood-pressure control. N Engl J Med.2015;373:2103-16.

8. Pierard LA. A randomized trial of intensive versus standard blood-pressure control. N Engl J Med.2016;23:2293.

9. Barrios V, Escobar C. New targets in arterial hypertension, are they justified? Rev Esp Cardiol. 2018;71(8):608-11.

10. Vipler B. A randomized trial of intensive versus standard blood-pressure control. N Engl J Med.2016;23:2290-1.

11. García-Zamora S, Botto F. Is the evidence provided by the SPRINT trial solid enough to support a systolic blood pressure threshold of 120 $\mathrm{mmHg}$ ? Rev Esp Cardiol.2019;72:520-1.

12. McCormick BB. A randomized trial of intensive versus standard blood-pressure control. N Engl J Med.2016;23:2291-2.

13. Esler M. SPRINT, or false start, toward a lower universal treated blood pressure target in hypertension. Hypertension.2016;67:266-7.

14. Touyz RM, Dominiczak AF. Successes of SPRINT, but still some hurdles to cross. Hypertension.2016;67:268-9.

15. Schiffrin EL, Calhoun DA, Flack JM. SPRINT proves that lower is better for nondiabetic high-risk patients, but at a price. Am J Hypertens.2016;29:2-4.

16. Saiz LC, Gorricho J, Garjón J, Celaya MC, Muruzábal L, Malón MDM, et al. Blood pressure targets for the treatment of people with hypertension and cardiovascular disease. Cochrane Database Syst Rev. 2017; 10:CD010315

17. Malhotra $R$, Nguyen HA, Benavente $O$, Mete M, Howard BV, Mant J, et al. Association between more intensive vs less intensive blood pressure lowering and risk of mortality in chronic kidney disease stages 3 to 5: A systematic review and meta-analysis. JAMA Intern Med. 2017:177:1498-505

18. Yusuf S, Lonn E. The SPRINT and the HOPE-3 trial in the context of other blood pressure-lowering trials. JAMA Cardiol.2016;1:857-8.

19. Schünemann $H$, Brożek J, Guyatt $G$, Oxman A. Manual GRADE para calificar la calidad de la evidencia y la fuerza de la recomendación (1 ${ }^{\text {a }}$ ed. en español). Bogotá, Colombia: Pontificia Universidad Javeriana, Facultad de Medicina, Departamento de Epidemiología y Bioestadística; 2017.

20. Moher D, Liberati A, Tetzlaff J, Altman DG; PRISMA Group. Preferred reporting items for systematic reviews and meta-analyses: the PRISMA statement. PloS Med.2009;6:e1000097.

21. Higgins JPT, Green S, editores. Cochrane Handbook for Systematic Reviews of Interventions. Version 5.0.2. The Cochrane Collaboration ${ }^{\circledR}$; 2008.

22. Higgins JPT, Altman DG, Gøtzsche PC, Jüni $P$, Moher D, Oxman AD, et al. The Cochrane Collaboration's tool for assessing risk of bias in randomised trials. BMJ. 2011;343:d5928.

23. Sterne JAC, Savović J, Page MJ, Elbers RG, Blencowe NS, Boutron I, et al. Rob 2: a revised tool for assessing risk of bias in randomised trials. BMJ. 2019;366:14898.

24. Dersimonian R, Laird N.Meta-analysis in clinical trials. Control Clin Trials.1986;7:177-88.

25. Higgins JPT, Thompson SG, Deeks JJ, Altman DG. Measuring inconsistency in meta-analyses. BMJ.2003;327:557-60.

26. ACCORD Study Group, Cushman WC, Evans GW, Byington RP Goff DC Jr, Grimm RH Jr, Cutler JA, et al. Effects of intensive blood-pressure control in type 2 diabetes mellitus. N Engl J Med.2010;362:1575-85.

27. Lonn EM, Bosch J, López-Jaramillo $P$, Zhu J, Liu L, Pais $P$, et al Blood-pressure lowering in intermediate-risk persons without cardiovascular disease.N Engl J Med.2016;374:2009-20.

28. SPS3 Study Group, Benavente OR, Coffey CS, Conwit R, Hart RG, McClure LA, Pearce LA, et al. Blood-pressure targets in patients with recent lacunar stroke: the SPS3 randomised trial. Lancet. 2013;382:507-15.

29. Lewington S, Clarke R, Qizilbash N, Peto R, Collins R. Prospective Studies Collaboration. Age-specific relevance of usual blood pressure to vascular mortality: a meta-analysis of individual data for one million adults in 61 prospective studies. Lancet.2002;360:1903-13. 
30. Ettehad D, Emdin CA, Kiran A, Anderson SG, Callender T, Emberson J et al. Blood pressure lowering for prevention of cardiovascular disease and death: a systematic review and meta-analysis. Lancet, 2016;387:957-67.

31. Bangalore S, Toklu B, Gianos E, Schwartzbard A, Weintraub H, Ogedegbe G, et al. Optimal systolic blood pressure target after SPRINT: Insights from a network meta-analysis of randomized trials. Am J Med.2017;130:707-19.

32. Thomopoulos C, Parati G, Zanchetti A. Effects of blood pressure lowering on outcome incidence in hypertension: 7. Effects of more vs. less intensive blood pressure lowering and different achieved blood pressure levels - updated overview and meta-analyses of randomized trials. J Hypertens.2016:34:613-22.

33. Sundström J, Arima H, Jackson R, Turnbull F, Rahimi K, Chalmers J, et al. Effects of blood pressure reduction in mild hypertension: a systematic review and meta-analysis. Ann Intern Med.2015;162:184-91.

34. Bundy JD, Li C, Stuchlik P, Bu X, Kelly TN, Mills KT, et al. Systolic blood pressure reduction and risk of cardiovascular disease and mortality: A systematic review and network meta-analysis.JAMA Cardiol. 2017;2:775-81.
35. Blood Pressure Lowering Treatment Trialists' Collaboration. Blood pressure-lowering treatment based on cardiovascular risk: a meta-analysis of individual patient data. Lancet.2014;384:591-8.

36. Thomopoulos C, Parati G, Zanchetti A. Effects of blood pressure lowering on outcome incidence in hypertension: 3 . Effects in patients at different levels of cardiovascular risk--overview and meta-analyses of randomized trials. J Hypertens.2014;32:2305-14.

37. Karmali KN, Lloyd-Jones DM, van der Leeuw J, Goff DC Jr, Yusuf S, Zanchetti $A$, et al. Blood pressure-lowering treatment strategies based on cardiovascular risk versus blood pressure: A meta-analysis of individual participant data. PLoS Med. 2018;15:e1002538.

38. Xie X, Atkins E, Lv J, Bennett A, Neal B, Ninomiya T, et al. Effects of intensive blood pressure lowering on cardiovascular and renal outcomes: updated systematic review and meta-analysis. Lancet.2016:387:435-43.

39. Brunström M, Carlberg B. Association of blood pressure lowering with mortality and cardiovascular disease across blood pressure levels: A systematic review and meta-analysis. JAMA Intern Med.2018;178:28-36.

40. Williams B, Mancia G, Spiering W, Agabiti Rosei E, Azizi M, Burnier M,et al. 2018 ESC/ESH Guidelines for the management of arterial hypertension. Eur Heart J. 2018;39(33):3021-104 


\section{Beneficios y riesgos potenciales de las metas intensivas en el tratamiento de la hipertensión arterial. Revisión sistemática y metaanálisis de ensayos clínicos}

\section{Benefits and potential risks of intensive goals in the treatment of arterial hypertension. Systematic review and meta-analysis of clinical trials}

Sebastián García-Zamora*, Andrés Rosende, Brunilda Casetta, María F. Grande-Ratti, Natalia Carli, Maria F. Bertarini, María A. Alcuaz y Verónica Schoj

Dirección Nacional de Promoción de la Salud y Control de Enfermedades Crónicas No Transmisibles, Ministerio de Salud de la Nación Argentina,Buenos Aires, Argentina

\section{Material Suplementario}

\section{Anexo: Evaluación del riesgo de sesgo de cada estudio particular}

\begin{tabular}{|l|l|l|}
\hline \multicolumn{3}{|c|}{ Estudio ACCORD ${ }^{26}$} \\
\hline Sesgo & $\begin{array}{l}\text { Juicio de los } \\
\text { autores }\end{array}$ & Comentarios adicionales \\
\hline $\begin{array}{l}\text { Generación de } \\
\text { secuencia aleatoria }\end{array}$ & bajo riesgo & \\
\hline Asignación oculta & bajo riesgo & \\
\hline $\begin{array}{l}\text { Cegamiento del } \\
\text { personal y los } \\
\text { participantes }\end{array}$ & alto riesgo & Estudio abierto \\
\hline $\begin{array}{l}\text { Cegamiento de los } \\
\text { evaluadores }\end{array}$ & bajo riesgo & \\
\hline $\begin{array}{l}\text { Evaluación } \\
\text { incompleta de datos } \\
\text { de resultado }\end{array}$ & bajo riesgo & \\
\hline Reporte selectivo & bajo riesgo & \\
\hline Otros sesgos & riesgo incierto & $\begin{array}{l}\text { Los autores no proveen } \\
\text { información respecto del } \\
\text { abandono del estudio por } \\
\text { efectos adversos al } \\
\text { tratamiento farmacológico }\end{array}$ \\
\hline
\end{tabular}

\begin{tabular}{|l|l|l|}
\hline \multicolumn{2}{|c|}{ Estudio HOPE $3^{27}$} \\
\hline Sesgo & $\begin{array}{l}\text { Juicio de } \\
\text { los autores }\end{array}$ & Comentarios adicionales \\
\hline $\begin{array}{l}\text { Generación de } \\
\text { secuencia } \\
\text { aleatoria }\end{array}$ & bajo riesgo \\
\hline $\begin{array}{l}\text { Asignación } \\
\text { oculta }\end{array}$ & bajo riesgo \\
\hline $\begin{array}{l}\text { Cegamiento del } \\
\text { personal y los } \\
\text { participantes }\end{array}$ & bajo riesgo \\
\hline $\begin{array}{l}\text { Cegamiento de } \\
\text { los evaluadores }\end{array}$ & bajo riesgo \\
\hline $\begin{array}{l}\text { Evaluación } \\
\text { incompleta de } \\
\text { datos de } \\
\text { resultado }\end{array}$ & bajo riesgo & \\
\hline $\begin{array}{l}\text { Reporte selectivo } \\
\text { Otros sesgos }\end{array}$ & bajo riesgo & riesgo \\
incierto & $\begin{array}{l}\text { Los autores no discriminan } \\
\text { específicamente la ocurrencia } \\
\text { de efectos adversos serios }\end{array}$ \\
\hline
\end{tabular}

Correspondencia:

*Sebastián García-Zamora

E-mail: sebagz83@gmail.com
Disponible en internet: 03-09-2020 Arch Cardiol Mex. 2020;90(4):1-5 www.archivoscardiologia.com 405-9940 / @ 2020 Instituto Nacional de Cardiología Ignacio Chávez. Publicado por Permanyer. Este es un artículo open access bajo la licencia CC BY-NC-ND (http://creativecommons.org/licenses/by-nc-nd/4.0/). 


\begin{tabular}{|c|c|c|}
\hline \multicolumn{3}{|c|}{ Estudio SPRINT $^{7}$} \\
\hline Sesgo & $\begin{array}{l}\text { Juicio de los } \\
\text { autores }\end{array}$ & Comentarios adicionales \\
\hline $\begin{array}{l}\text { Generación de } \\
\text { secuencia aleatoria }\end{array}$ & bajo riesgo & \\
\hline Asignación oculta & riesgo incierto & $\begin{array}{l}\text { Los autores no } \\
\text { declararon como } \\
\text { llevaron adelante este } \\
\text { proceso }\end{array}$ \\
\hline $\begin{array}{l}\text { Cegamiento del } \\
\text { personal y los } \\
\text { participantes }\end{array}$ & alto riesgo & Estudio abierto \\
\hline $\begin{array}{l}\text { Cegamiento de los } \\
\text { evaluadores }\end{array}$ & bajo riesgo & \\
\hline $\begin{array}{l}\text { Evaluación } \\
\text { incompleta de datos } \\
\text { de resultado }\end{array}$ & bajo riesgo & \\
\hline Reporte selectivo & bajo riesgo & \\
\hline Otros sesgos & alto riesgo & $\begin{array}{l}\text { Detención prematura del } \\
\text { estudio }\end{array}$ \\
\hline
\end{tabular}

\begin{tabular}{l|l|l|}
\hline \multicolumn{3}{|c|}{ Estudio SPS-3 ${ }^{28}$} \\
\hline Sesgo & $\begin{array}{l}\text { Juicio de } \\
\text { los autores }\end{array}$ & Comentarios adicionales \\
\hline $\begin{array}{l}\text { Generación de } \\
\text { secuencia } \\
\text { aleatoria }\end{array}$ & bajo riesgo \\
\hline $\begin{array}{l}\text { Asignación } \\
\text { oculta }\end{array}$ & bajo riesgo & \\
\hline $\begin{array}{l}\text { Cegamiento del } \\
\text { personal y los } \\
\text { participantes }\end{array}$ & alto riesgo & Estudio abierto \\
\hline $\begin{array}{l}\text { Cegamiento de } \\
\text { los evaluadores }\end{array}$ & bajo riesgo & \\
\hline $\begin{array}{l}\text { Evaluación } \\
\text { incompleta de } \\
\text { datos de } \\
\text { resultado }\end{array}$ & alto riesgo & $\begin{array}{l}\text { Los autores no declararon a que } \\
\text { rama pertenecían los pacientes } \\
\text { que se perdieron del seguimiento } \\
\text { (3\%) ni aquellos que terminaron } \\
\text { el mismo prematuramente (15\%) }\end{array}$ \\
\hline $\begin{array}{l}\text { Reporte } \\
\text { selectivo }\end{array}$ & $\begin{array}{l}\text { riesgo } \\
\text { incierto }\end{array}$ & $\begin{array}{l}\text { Reporte incompleto de efectos } \\
\text { adversos relacionados con el } \\
\text { tratamiento antihipertensivo }\end{array}$ \\
\hline $\begin{array}{l}\text { Otros sesgos } \\
\text { bajo riesgo }\end{array}$ & \\
\hline
\end{tabular}


Suplementaria Figuras

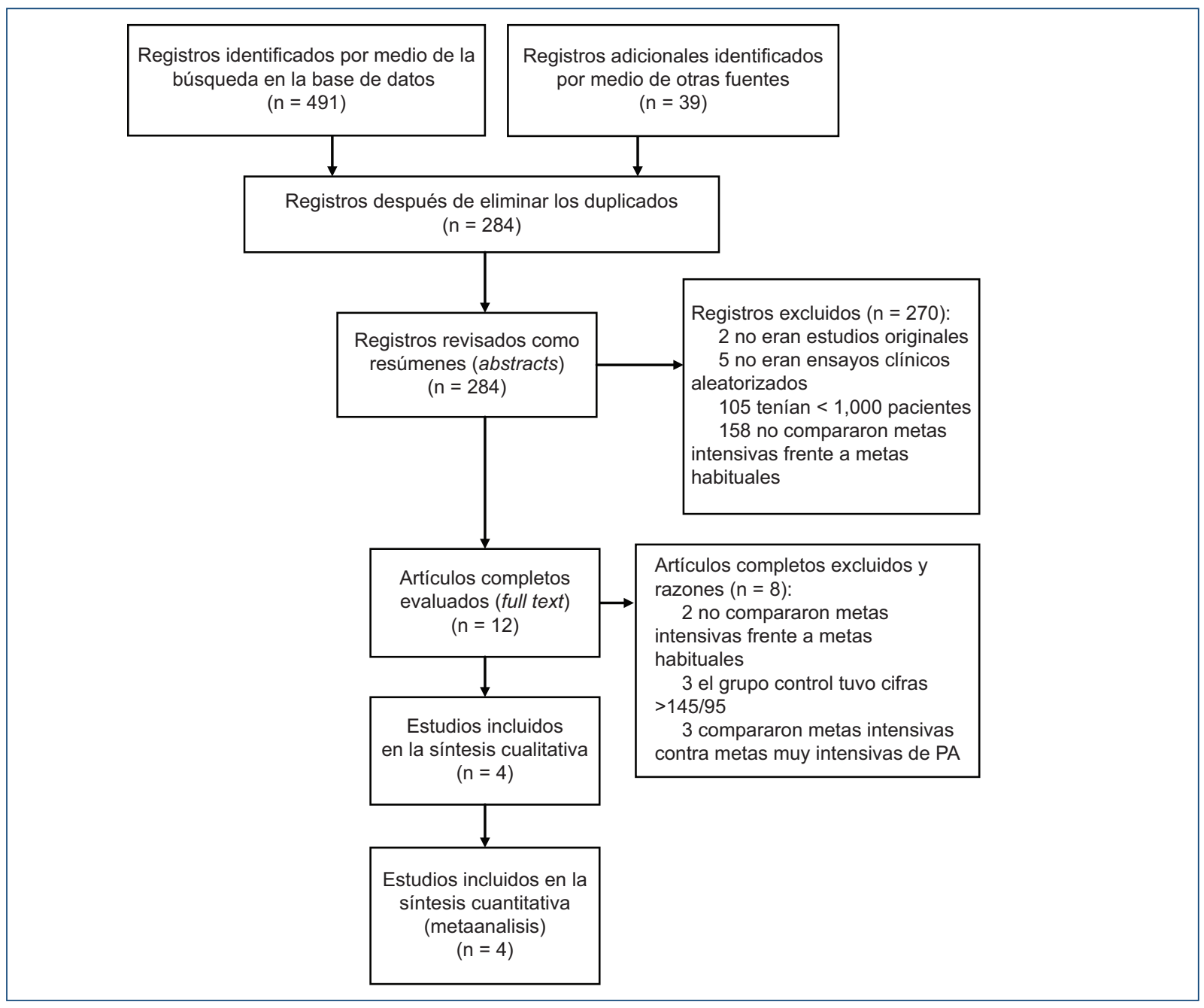

Figura suplementaria 1. Flow chart. 


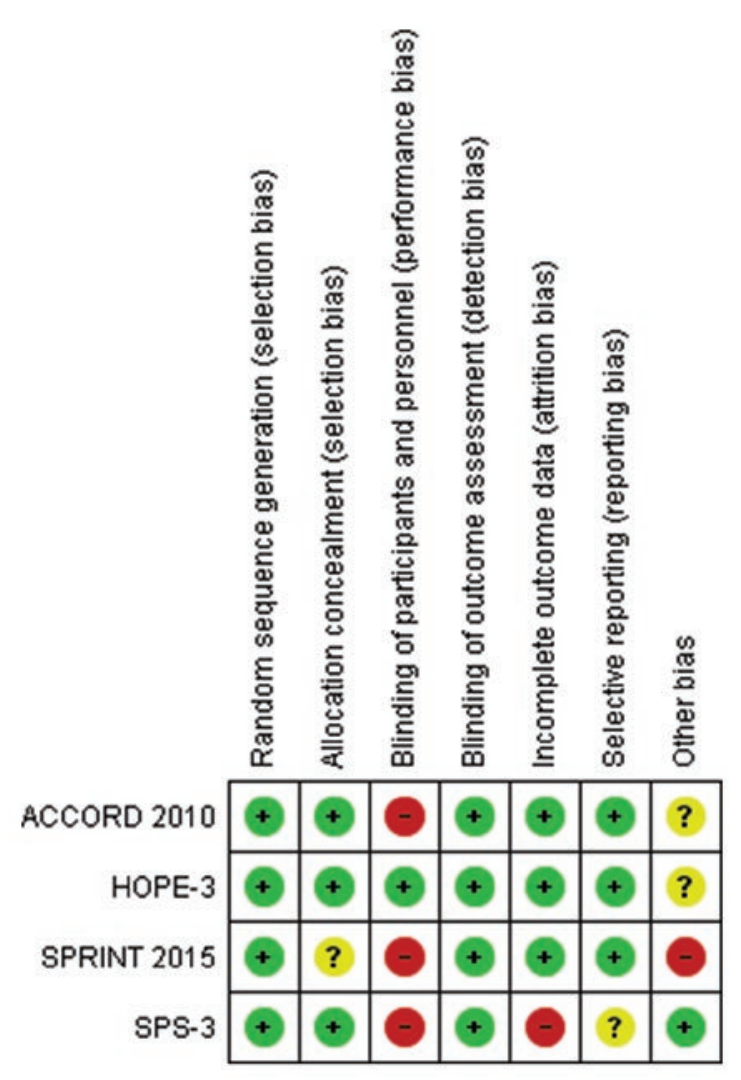

Figura suplementaria 2. Riesgo de sesgo de los estudios incluidos: el juicio establecido es opinión de los autores del presente metaanálisis. Los círculos verdes implican un "bajo riesgo de sesgo", los círculos amarillos un "riesgo incierto" y los rojos un «riesgo alto» de sesgo en el dominio correspondiente.

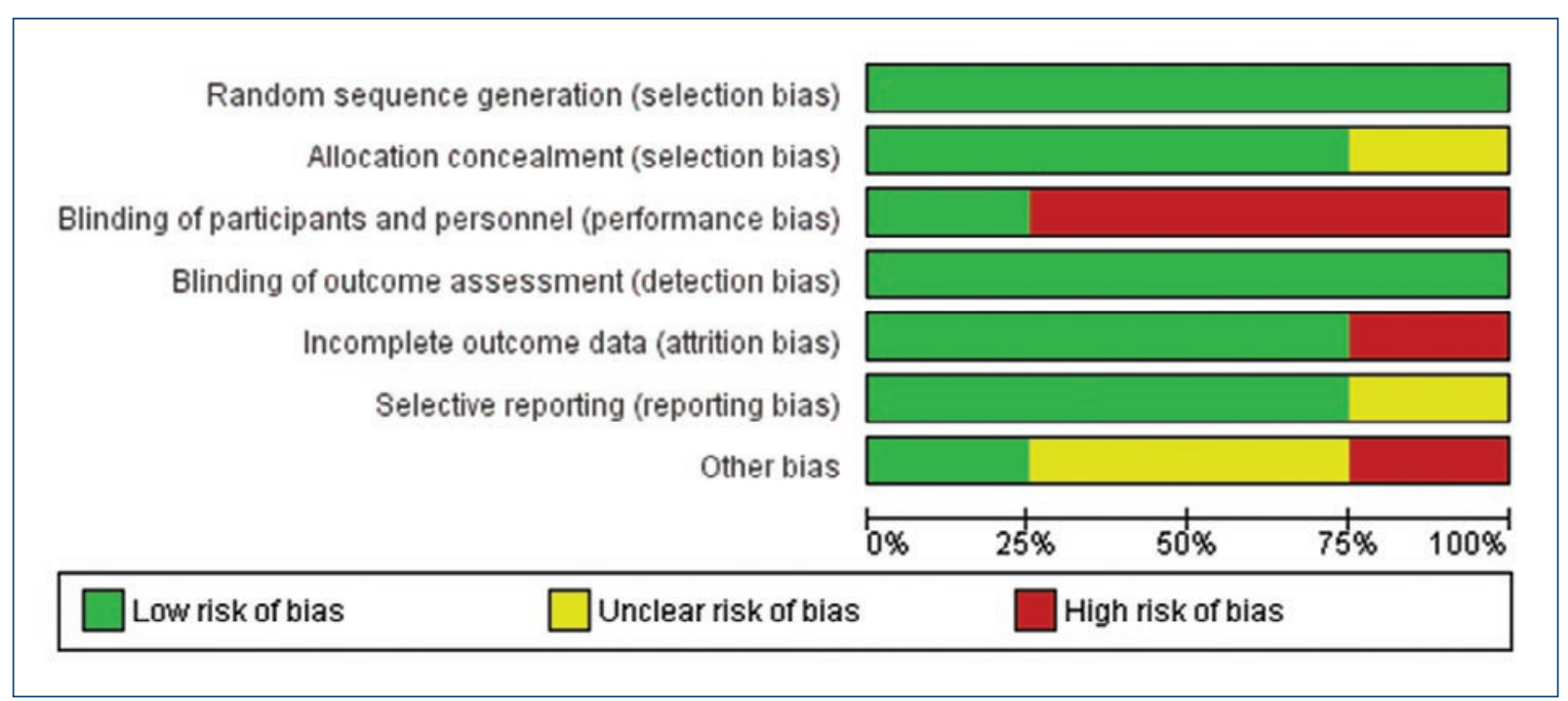

Figura suplementaria 3. Ponderación global del riesgo de sesgos según dominios. 


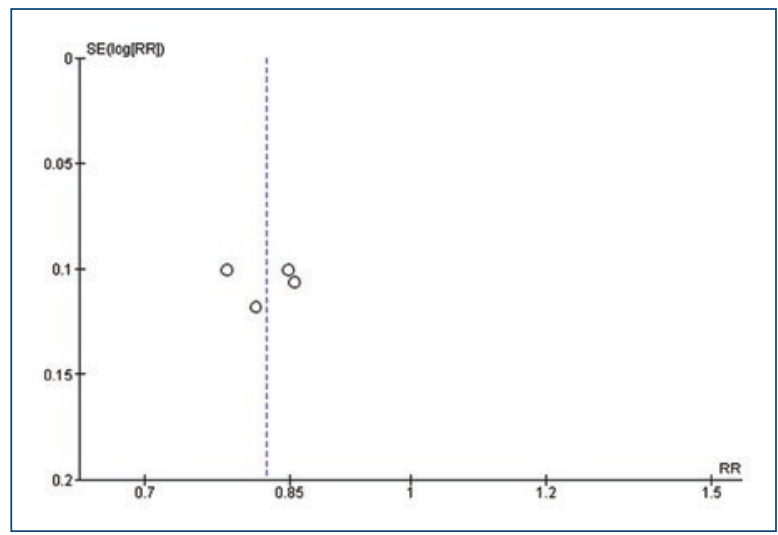

Figura suplementaria 4. Gráfico de embudo (funnel plot) para sesgo de publicación. 